\title{
Current Treatment Options for Local Residual Nasopharyngeal Carcinoma
}

\author{
S. D. Stoker, $M D^{1}$ \\ J. N. A. van Diessen, $M D^{2}$ \\ J. P. de Boer, dr $M D^{3}$ \\ B. Karakullukcu, $M D^{1}$ \\ C. R. Leemans, prof. dr $M D^{4}$ \\ I. B. Tan, prof. $d r M D^{1, *}$
}

\author{
Address \\ $1, \star$ Department of Head and Neck Surgery and Oncology, The Netherlands \\ Cancer Institute, Plesmanlaan 121, Amsterdam, The Netherlands \\ Email: i.tan@nki.nl \\ ${ }^{2}$ Department of Radiotherapy, The Netherlands Cancer Institute, Plesmanlaan \\ 121, Amsterdam, The Netherlands \\ ${ }^{3}$ Department of Hemato-oncology, The Netherlands Cancer Institute, \\ Plesmanlaan 121, Amsterdam, The Netherlands \\ ${ }^{4}$ Department of otolaryngology/head and neck surgery, VU University Medical \\ Center Amsterdam, Boelelaan 1117, Amsterdam, The Netherlands
}

Published online: 16 November 2013

(C) The Author(s) 2013. This article is published with open access at Springerlink.com

Keywords Nasopharyngeal carcinoma - Residual disease - Persistent disease - Local failure - Reirradiation . Brachytherapy - Stereotactic radiotherapy - Surgery - Photo dynamic therapy - Chemotherapy

\section{Opinion statement}

Local residual disease occurs in 7-13\% after primary treatment for nasopharyngeal carcinoma (NPC). To prevent tumor progression and/or distant metastasis, treatment is indicated. Biopsy is the "gold standard" for diagnosing residual disease. Because late histological regression frequently is seen after primary treatment for NPC, biopsy should be performed when imaging or endoscopy is suspicious at 10 weeks. Different modalities can be used in the treatment of local residual disease. Interestingly, the treatment of residual disease has better outcomes than treatment of recurrent disease. For early-stage disease (rT1-2), treatment results and survival rates are very good and comparable to patients who had a complete response after the first treatment. Surgery (endoscopic or open), brachytherapy (interstitial or intracavitary), external or stereotactic beam radiotherapy, or photodynamic therapy all have very good and comparable response rates. Choice should depend on the extension of disease, feasibility of the treatment, and doctor's and patient's preferences and experience, as well as the risks of the adverse events. For the more extended tumors, choice of treatment is more difficult, because complete response rates are poorer and severe side effects are 
not uncommon. The results of external beam reirradiation and stereotactic radiotherapy are better than brachytherapy for T3-4 tumors. Photodynamic therapy resulted in good palliative responses in a few patients with extensive disease. Also, chemotherapeutics or the Epstein-Barr virus targeted therapies can be used when curative intent treatment is not feasible anymore. However, their advantage in isolated local failure has not been well described yet. Because residual disease often is a problem in countries with a high incidence of NPC and limited radiotherapeutic and surgical facilities, it should be understood that most of the above mentioned therapeutic modalities (radiotherapy and surgery) will not be readily available. More research with controlled, randomized trials are needed to find realistic treatment options for residual disease.

\section{Introduction}

Treatment results of nasopharyngeal carcinoma (NPC) have been improved by the introduction of combining chemotherapy and radiotherapy, better radiotherapy techniques, and enhanced imaging studies. Nevertheless, local failure occurs in $10 \%$ to $30 \%[1,2]$. Treatment of local failure is challenging, due to the anatomical difficult accessibility of the nasopharynx and the high risk on therapy related complications. Treatment options include surgery, reirradiation by external beam, brachytherapy or stereotactic radiotherapy, photodynamic therapy, and chemotherapy. Sometimes combinations are used. Many studies are conducted, but no consensus has been reached on the best treatment option for local failure. For earlystage residual disease (rT1-2), a significant benefit is found when salvage treatment is performed. Radiation and surgical modalities have comparable response, survival, and complication rates. For more advanced tumors (rT3-4), the benefit of salvage treatment is less clear, probably due to the high treatment related morbidity and mortality, caused by large tumor volumes and involvement of surrounding structures $[2,3 \bullet]$.

Local failure can be divided in residual or recurrent disease. The definition of residual disease is arbitrary. Usually it is defined as the confirmation of disease occurring within 6 months after treatment. When disease is found after these 6 months, provided that previous complete response was seen, it is defined as recurrent disease. The incidence of residual disease is 7-13\% [412]. In reviews of treatment options for local failure of NPC, residual or recurrent disease rarely is distinguished. Articles that have made this distinction found that residual disease responds better than recurrent disease to the modalities based on reirradiation
$[13,14]$. This is controversial, because studies on reirradiation for recurrent head and neck tumors have shown that a longer interval between the initial and the second course of radiation is an independent prognostic factor for a more beneficial treatment outcome [15]. In view of that, there might be a difference in the preferential treatment for residual compared with recurrent NPC.

We would like to emphasize the need to study residual disease, because we believe, based on our experience in Indonesia, that residual disease is an underexposed problem $[16 \bullet \bullet, 17 \bullet]$. The reason for the good results of residual disease compared with recurrent might be derived from insufficient primary treatment. In many countries where NPC has high incidences (like Tunisia, Algeria, Philippines, Indonesia), access to high-standard medical care is not readily available for everyone [18•]. Unfortunately, scientific publications from these regions that could expose these problems also are lacking. Reasons for the high incidence of persistent disease are that treatment is frequently given by old fashioned and/or poorly maintained equipment, besides 3-D conventional radiotherapy or IMRT and also enhanced imaging facilities, such as MRI and PET scan are in most hospitals not available. In addition, studies in Indonesia revealed that patients have more advanced-stage disease due to delay in diagnosis and long intervals to treatment due to waiting lists for radiotherapy $[16 \bullet \bullet]$. Also, the overall treatment time is prolonged due to problems, such as unreliable equipment, public holidays, and poor physical condition of the patients [19]. For these patients, it is of great importance to find realistic treatment options for residual NPC. Additional treatment could in these 
patients be considered as a boost after the primary treatment aiming for a complete response.

To our knowledge, no review on solely residual disease has been published. The focus of this review is on the treatment of local residual disease after primary radiation or chemoradiation, given with curative intent. Indication, treatment procedure, outcome, and side effects will be compared per modality.

\section{Treatment}

- Treatment of primary NPC consists of full dose external beam radiation (66-70 Gy) to the nasopharynx and positive neck nodes and a prophylactic dose of 40-50 Gy to the rest of the neck. Chemotherapy can be administered as neoadjuvant, concurrent, or adjuvant. The current standard treatment is radiotherapy for stage I and concurrent chemoradiotherapy for Stage II-IVb. Stage IVc is treated with only palliative intent (AJCC 2010).

- After radiotherapy, it is hard to distinguish postirradiation effects from residual tumor by endoscopy and CT scan and/or MRI. PET may distinguish better, but biopsy remains the "gold standard." To date, there is much interest on Epstein-Barr Virus (EBV) markers for NPC, because the majority of NPC is EBV related, especially in endemic countries. Elevated DNA load and antibodies for EBV in blood are correlated to the presence of NPC and valuable in detecting NPC recurrences [20]. Unfortunately, the positive predicting value of elevated levels directly after therapy is limited. Besides, these tests do not give information on the location of the tumor. Hence, they are not yet adequate in confirming who needs additional treatment for local failure. A brush for measuring EBV load in the nasopharynx might have more potential for confirming local residual disease [21, 22]. However, more research for its use as a determinant for giving adjuvant treatment is needed.

- The optimal length of the interval to retreatment of NPC is a topic of debate. Some investigators advocate treating as soon as possible in order to prevent progression and dissemination, others advocate waiting for at least 10 weeks, because late histological regressions might occur. Kwong et al. [23] studied the histological regression and performed serial posttreatment-nasopharyngeal biopsies in 803 patients. After treatment, $77 \%$ of the biopsies were negative, $4 \%$ regressed within 5 weeks, and $12 \%$ regressed between weeks 5 and 12. In $7 \%$, the biopsies remained tumor-positive at 12 weeks. Hence, $70 \%$ of the posttreatment-positive biopsies regressed within 12 weeks without additional treatment. A curve, containing the percentages of regression per posttreatment week, reaches a plateau after the tenth week, indicating that after week 10 the chances for spontaneous regression diminish and justify additional treatment. When interpreting treatment results for persistent disease, one 
should keep in mind these findings, because results might be biased by the spontaneous regression of late-responding tumors.

- Kwong et al. also studied the tumor control rates for the different regression patterns [23]. Early regression was defined as negative biopsy before the fifth week, late regression as regression between weeks 5 and 12, and persistent disease as a positive biopsy at 12 weeks. There was no difference between the late and early regression in 5-year local control rate ( $82 \%$ vs. $77 \%)$, regional and distant metastasis-free rate (69 \% vs. $72 \%$ ) and overall survival (75\% vs. $79 \%$ ). This justifies a wait-and-see policy for positive biopsies until the tenth week. Patients with persistent disease at 12 weeks had significantly lower local control rate (40\%), regional and distant metastasis-free rate (47\%), and overall survival (54\%). Hence, additional treatment for this group is indicated.

- Different treatment modalities can be used for the treatment of residual NPC. The extent of the disease, comorbidity of the patient, initial treatment, availability of treatment modalities, and experience of the treating surgeon and radiation oncologist are decisive in the choice of treatment.

- The choice for reirradiation in recurrent or persistent head and neck tumors after a previous full radiation course should be considered carefully. A higher cumulative radiation dose gives more chance on tumor control but more risk to complication. Moreover, a shorter interval between previous radiation, larger tumor volume, and poor clinical condition are associated with a worse outcome and more complications. Only for a limited patient group, who have no other curative options or as adjuvant treatment to surgery, reirradiation of the head and neck can be considered [24, 25]. For NPC, different irradiation modalities have been used, such as brachytherapy, stereotactic radiation, and external beam reirradiation. Contrary to other head and neck tumors and recurrent NPC, the results of reirradiation for residual NPC are quite good.

\section{Procedures}

- Brachytherapy delivers a high radiation dose to the target area with a steep dose curve to avoid a high dose to surrounding tissue. Brachytherapy has been used for NPC since the 1920s [13]. For residual NPC, an applicator (intracavitary brachytherapy (ICB)) or interstitial gold implantations have been used. Studies on solely residual disease are limited. Table 1 shows the results and side effects of these studies. 
- The advantage of intracavitary brachytherapy (ICB) is that the applicator can be inserted under local anesthesia. Tumors confined to the mucosal or submucosal nasopharynx are suitable. This treatment is not indicated for tumors extending to the parapharyngeal site, base of skull, or oropharynx. To prevent failure due to geographical miss, sophisticated imaging, such as CT scan or MRI, should be performed.

- Teo et al. published one of the largest series of ICB for residual NPC [7]. Administration of $24 \mathrm{~Gy} / 3$ fractions/15 days to 71 patients with a positive biopsy after 6 weeks resulted in a complete response in $93 \%$. Five-year local control (LC) of these patients was $74 \%$ and overall survival (OS) was $68 \%$.

- Law et al. [26] treated 23 patients with ICB for residual (rT1-2) disease, using 25-50 Gy in 4-7 days. Complete response (CR) was obtained in $97 \%$ and the 5-year LC was with $90 \%$.

- Leung et al. [6] treated 87 patients with ICB (22.5-24 Gy in 3 weeks). If the nasopharynx was suspected on endoscopy, a biopsy was performed at a median of 6 weeks posttreatment. The results were compared to patients who had a complete response after primary treatment with EBRT only. Patients treated for earlystage residual disease had equal local control rates as patients who had a complete response after primary EBRT (80-95 \%). The authors postulated that ICB might be effective to compensate for inadequate primary treatment. Patients who had advanced T stage at primary diagnosis did worse both with respect to local control and survival. A substantial number of these patients might still have had extension outside the nasopharynx. A different treatment modality might have been more suitable for this group. Hence, these results confirm the benefit of restaging before retreatment.

- Kwong et al. [13] used brachytherapy with radioactive gold implants for residual disease. Small lesions with clear boundaries or lesions situated on one side of the nasopharynx were suitable. Lesions in the eustachian tube or extending into the nasal cavity were not suitable, because the implants cannot be attached firmly to thin mucosa. Treatment was not performed within 3 months after primary radiation. Gold implants were inserted under general anesthesia, via an incision through the soft palate. A dose of $60 \mathrm{~Gy}$ at $0.5 \mathrm{~cm}$ away from the source was prescribed. The median number of implants was six. Patients were discharged on the fourth postoperative day. Persistent disease did better than recurrent disease (LC $87 \%$ vs. $63 \%$, OS $79 \%$ vs. $54 \%$ ). Some patients with parapharyngeal extension or skull base erosion also were treated, despite the fact they 


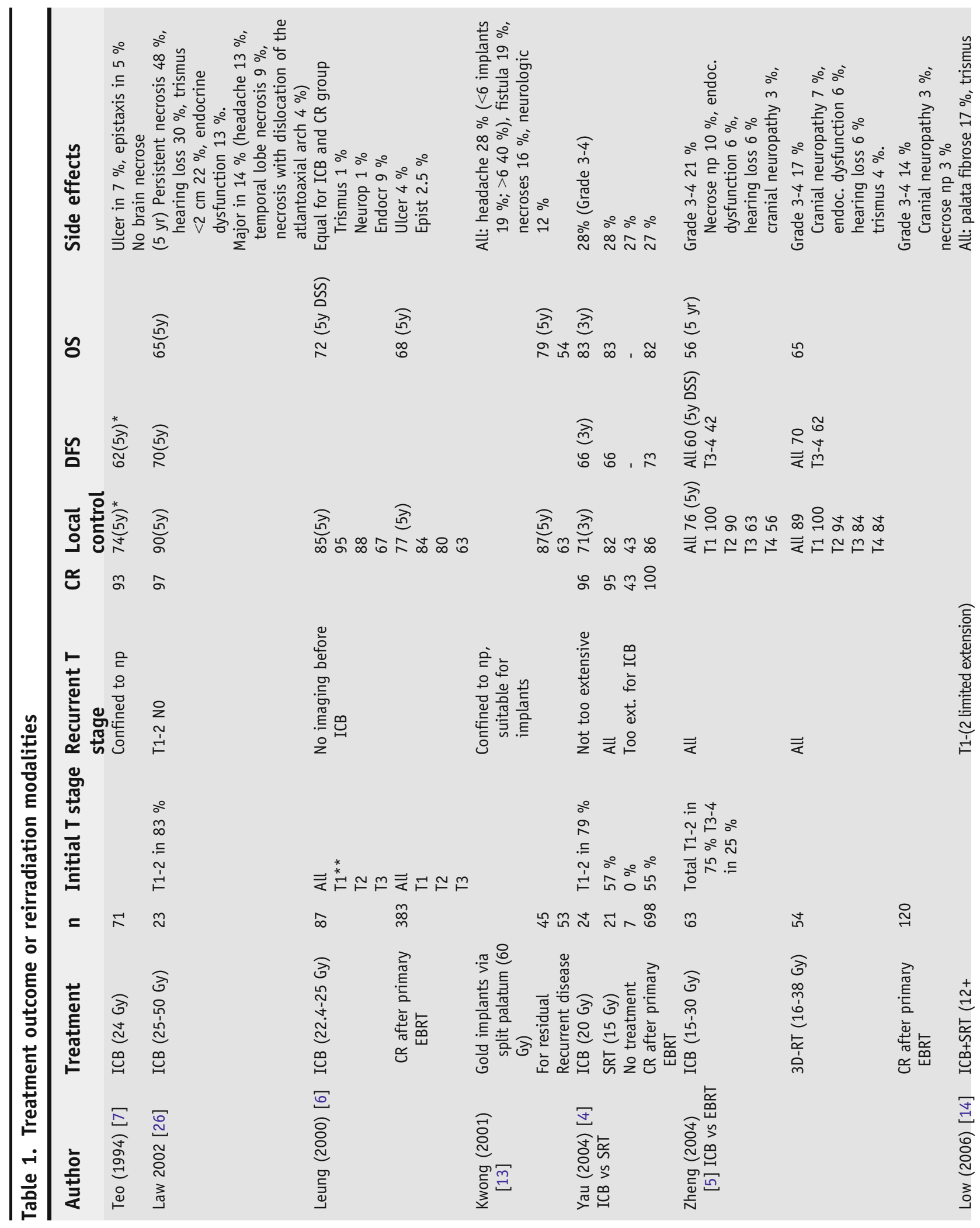




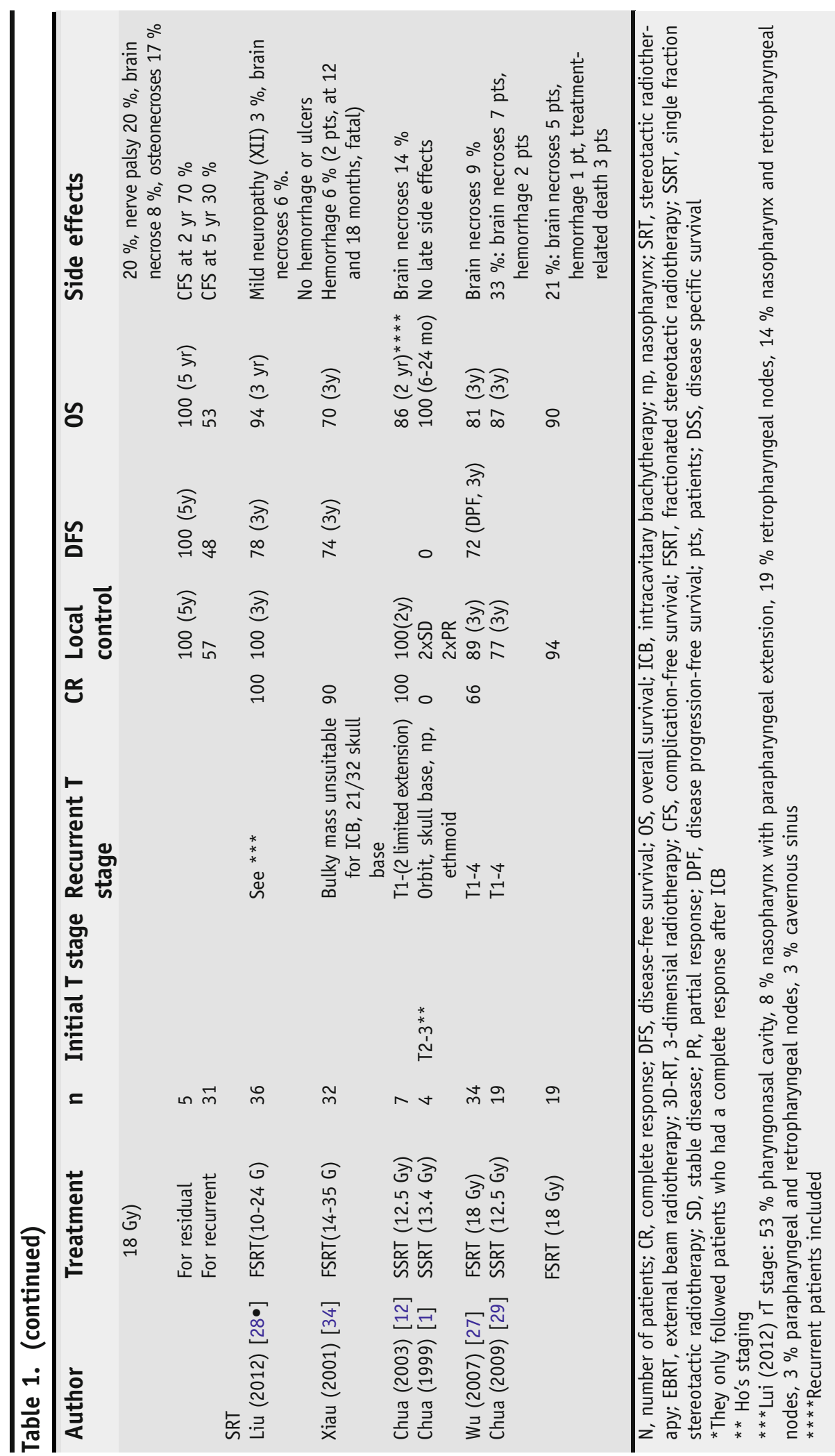


Stereotactic radiation

were not strictly eligible for this treatment. However, these patients got the benefit of the doubt, because confirmation of the disease by biopsy was not possible at these sides. As expected, these patients did worse than patients with disease confined to the nasopharynx on 5-year LC (52\% vs. $72 \%)$.

- Since 1991 stereotactic radiation (SRT) is used in the treatment of local failure of NPC [27]. SRT can be delivered as a single dose or in multiple fractions.

- Most studies are retrospective and patient selection often is based on the unsuitability for brachytherapy or nasopharyngectomy. Tumors suitable for SRT can be lesions with skull base erosion or orbit invasion or intracranial extension. The CR and 3-year LC are up to $100 \%$ for T1-3 disease [28•]. In Table 1, the studies involving residual disease are shown.

- Fractionated versus single fraction stereotactic radiation; based on the biological principles of radiation, fractionated treatment would provide a favorable therapeutic ratio with fewer side effects. Chua and $\mathrm{Wu}$ et al. compared their treatment results in a matched cohort analysis [29]. Patients were matched individually for the following prognostic factors: persistent versus recurrent disease, rT stage, and tumor volume. Fractionated SRT had a better outcome in 3-year local failure-free survival, especially for recurrent disease and tumors growing beyond the nasopharynx (T2-4). The subgroup analysis for persistent disease showed no significant benefit for either fractionated or single fraction SRT.

- Yan et al. $[11,30]$ conducted two large studies concerning the treatment of residual disease by EBRT. The first study was retrospective and consisted of 182 patients (20-50 Gy); the second was a prospective study with 78 patients $(20 \mathrm{~Gy}, 4 \mathrm{~Gy} / \mathrm{fr}, 2 \mathrm{fr} /$ week). Both studies involved patients treated and untreated for residual disease and found that treated patients did better with respect to LC and OS (Table 1). In the prospective study, these patients were compared with patients who had a complete response after the initial treatment. There was no difference in 3year LC (96\% and $94 \%)$ for the initial complete responders and the salvage group. The observation group with a positive biopsy did significantly worse (LC $64 \%$ ). OS was similar in all groups. Although there might be a bias, because biopsy was taken 1014 days after initial treatment, these studies emphasised the need for treatment of residual disease.

- Intensity-modulated EBRT (IMRT) has good results for the treatment of recurrent disease (5-year LC of $85-66 \%$ and OS of 71- 


\section{Comparison between modalities}

$30 \%$, depending on rT stage) $[31,32,33 \bullet]$. Unfortunately, most studies have a gap interval to reirradiation of more than 6 months. Because good results of other reirradiation techniques for residual disease have been achieved, IMRT also has the potential to be a good treatment for residual disease, but studies have to confirm this.

ICB vs 3D-RT In 2004, Zheng et al. [5] retrospectively compared the outcome of 3DRT and ICB for residual disease. Residual disease was confirmed by biopsy 2-8 weeks after initial treatment. ICB was given to 63 patients (15-30 Gy, depending on the gap period of radiation, 5-6 Gy/fr, $2 \mathrm{fr} /$ week). Staging was performed at diagnosis only. In the $3 \mathrm{D}$ group, 54 patients were included, CT imaging was performed for restaging, and all rT stages were included. The target volume was the tumor plus 5$\mathrm{mm}$ margin. Depending on the gap period, a dose of 16-38 Gy was given ( $2 \mathrm{~Gy} / \mathrm{fr}, 1 \mathrm{fr} /$ day). The 5-year overall survival and local failurefree survival for the 3D and the ICT groups were $65 \%$ vs. $56 \%$ and $89 \%$ vs. $76 \%$ (not significant). Subgroup analysis showed that initial T1-2 did better than T3-4 for both groups (LC $>90 \%$ vs. 56-84\%). For initial T1-2, no preferential treatment was found. For T3-4 tumors, the local failure-free survival was significantly better for the $3 \mathrm{D}$ group (84\% vs. $61 \%)$.

ICB vs SRT Yau et al. [4] retrospectively compared ICB with SRT for residual disease; 24 were treated by ICB (10-24 Gy 2 fr/week) and 21 with SRT (entire nasopharyngeal mucosa and gross residual tumor within 3$5 \mathrm{~mm}$ margin, $15 \mathrm{~Gy}$ ). The initial diagnosis was T3-4 in $21 \%$ of the ICB group and in $43 \%$ of the SRT group. The time interval to retreatment was 8-16 weeks. ICB and SRT had similar complete response rates (96\% and $95 \%$ ). Treatment results were compared to patients with a complete response after the primary treatment and an identical 3-year overall survival (82-83\%) was found. Local control rate was $84 \%$ in the initial complete responders, $71 \%$ in the ICB group, $82 \%$ in the SRT group, and $43 \%$ in the group of untreated patients with residual disease. Only the SRT group achieved local control rates comparable with the complete responders, despite the higher frequency of T3-4 disease at initial diagnosis. The incidence of grade 3-4 side effects were similar in the retreated patients and the group without salvage treatment.

\section{Complications}

- Adverse events commonly are seen after reirradiation and are the limiting factor in treatment (Table 1). After ICB, 12-28\% experience severe adverse events. Nasopharyngeal necrosis (4-10\%), endocrine dysfunction (6-9 \%), hearing loss (6-30\%), and cranial neuropathy $(1-12 \%)$ are frequently seen $[4-7,26]$. Law et al. [26] all gave the highest radiation dose and had the best LR rates for ICB; unfortunately they also had the most severe adverse events, e.g., temporal lobe necrosis was seen in $9 \%$ of the treated patients. Leung, Zheng, and Yau et al. [4-6] compared patients who had ICB with patients 
who got no additional treatment and found no significant differences in the number of adverse events. Brachytherapy with gold implants have comparable adverse events to ICB. Headache frequently is seen and related to the number of implants. Fistulas were seen in $50 \%$ of the patients, but by changing the palatal closing procedure into three layers they were not seen anymore. SRT caused brain necrosis in 9-25\% and severe haemorrhage in 6-8\%, in some cases leading to death $[29,34]$. Fractionated SRT should in theory give less adverse events, but the comparison made by Chua and $\mathrm{Wu}$ showed no differences with single fraction SRT. Risk for massive hemorrhage is present when there is tumor extension to the cavernous sinus, or retropharyngeally to the carotid sheet, or when there is mucosal necrosis, tumor progression, or carotid aneurysm. If the tumor encases the cavernous sinus or carotid artery, single-dose SRS should be avoided, but also multifraction SRT should be used very carefully [29]. Yan et al. [11] gave EBRT for residual disease and reported encephalomyelitis in $17 \%$ compared with $6 \%$ in the patients who only received primary EBRT only $(p<0.05)$. Comparable incidence rates of grade 3-4 side effects are mentioned after 3D-RT by Zheng et al. [5]. They also compared the results with patients who received primary EBRT only and found no significant difference in the incidence of side effect. Although the incidence of cranial neuropathy merits caution $(7 \%$ in the retreated group vs. $3 \%$ in the primary EBRTonly group). IMRT should in principle have fewer side effects than 2D and 3D EBRT. Unfortunately, no studies on residual disease only have been conducted.

\section{Special points}

- Brachytherapy, SRT, and EBRT are all suitable modalities for small residual lesions with comparable control and complication rates. When the tumor extends outside the nasopharynx, SRT or EBRT is preferable to brachytherapy. Based on the experience in recurrent disease, IMRT can be very effective in treating rT3-4 lesions, with acceptable side effect. Nevertheless, because residual disease has the highest incidence in regions where advanced techniques are not available and waiting lists for conventional EBRT exist, these treatment options are not realistic for these regions. For instance, in Indonesia 0.13 radiation units per 1,000,000 inhabitants are available compared with 2.5-5.5 units in European countries [35, 36]. In addition, in the countries with a high incidence of residual NPC often MRI facilities are not present, which is important in deciding the boundaries between tumor margins and healthy tissue to prevent side effects and have adequate tumor margins. These issues represent the problems when treating residual disease and the need to search for alternative and realistic options. 
- Most experience with nasopharyngectomy comes from countries with a very high incidence of nasopharyngeal carcinoma and with high standard medical care, such as China. Nasopharyngectomy can be performed by different approaches. The techniques are challenging and therefore should be performed by experienced hands only. Surgery can only be curative if the tumor mass is radically removed, hence there can not be intracranial extension, invasion of the base of the scull, or carotid artery involvement. Recently, more research on endoscopic surgery is performed, with promising results.

- The maxillary swing is an anterior-lateral approach [37•]. By a midfacial incision, extending over the mucosal of the hard palate, and osteotomies on the anterior maxilla, zygomatic arch, and hard palate, the maxilla on the side of the tumor can be swung laterally to expose the nasopharynx. One-sided tumors, with extension to lateral are the ideal tumors for this method. Extension to the contralateral side or oropharynx are less ideal. If there is extension or lymph node involvement in the parapharyngeal space, this also can be resected. Caution should be taken on the carotid artery. A free flap can be used when the base of the skull or the carotid artery is exposed.

- With the anterior approach, the midfacial degloving procedure, the maxilla is divided on both lateral walls and the nasopharynx is exposed from the front. Tumors in the midline and with only minimal lateral extension are best suited for this method, which is advantageous is that there is no facial incision and that the soft palate remains functional.

- The nasopharynx also can be entered from inferior, the transpalatal approach, whereby the palate is split, with or without a mandibular swing depending on the opening of the mouth. Tumors extending to the inferior nasopharynx or oral cavity with minimal extension to the sides are suitable for this method [38].

- Unfortunately, to our knowledge, no study has been published that included only persistent disease. Vlantis et al. [38] and Wei at al. [37•] both included residual disease patients in their studies without making a clear distinction between recurrent and residual disease. Both studies mention briefly that there were no differences between residual and recurrent disease with respect to local control or survival. In Table 2, results are shown for residual and recurrent disease together. In the following part, these studies will be outlined.

- Wei et al. [37•] performed the maxillary swing with curative intent on 246 patients, of which 37 had persistent disease. Patients with erosion at the skull base or invasion of the carotid artery were not included. During surgery, frozen sections were used. Radical margins were achieved in $78 \%$ and a 5 -year LC of $75 \%$. 


\begin{tabular}{|c|c|c|c|c|c|c|c|c|c|}
\hline Surgery & Treatment & n & $\begin{array}{l}\text { Initial T } \\
\text { stage }\end{array}$ & $\begin{array}{l}\text { Recurrent } \\
\text { T stage }\end{array}$ & CR & $\begin{array}{l}\text { Local } \\
\text { control }\end{array}$ & DFS & OS & Side effects \\
\hline $\begin{array}{l}\text { Wei }(2011) \\
{[37 \bullet]}\end{array}$ & $\begin{array}{c}\text { Maxillary } \\
\text { swing }\end{array}$ & 246 & & $\begin{array}{c}\text { No skull base } \\
\text { or carotid } \\
\text { invasion }\end{array}$ & 78 & 75 (5 yr) & 56 (5 yr) & & $\begin{array}{l}\text { Carotid bleeding } \\
\quad(1 \%) \\
\text { Palatal fistula } \\
(20 \%) \\
\text { Trismus }<2 \mathrm{~cm} \\
(21 \%)\end{array}$ \\
\hline \multirow{4}{*}{$\begin{array}{l}\text { Vlantis (2008) } \\
\text { [38] }\end{array}$} & Total & 80 & & * & $* *$ & LPFS (5 yr) & & rT1-3 (5y) & Not described \\
\hline & Transpalatal & 21 & $\begin{array}{l}\text { T1-2 } 57 \% \\
\text { T3-4 } 33 \%\end{array}$ & $\begin{array}{l}\text { T1-2 } 95 \% \\
>\text { T2 } 5 \%\end{array}$ & $\begin{array}{l}65 \\
-\end{array}$ & & & 43 & \\
\hline & $\begin{array}{c}\text { Maxillary } \\
\text { swing }\end{array}$ & 26 & $\begin{array}{l}\text { T1-2 } 73 \% \\
\text { T3-4 } 27 \%\end{array}$ & $\begin{array}{l}\text { T1-2 } 73 \% \\
>\text { T2 } 27 \%\end{array}$ & $\begin{array}{l}63 \\
-\end{array}$ & rT1-2 88 & & 59 & \\
\hline & $\begin{array}{l}\text { Facial } \\
\quad \text { degloving }\end{array}$ & 33 & $\begin{array}{l}\text { T1-2 } 76 \% \\
\text { T3-4 } 21 \%\end{array}$ & $\begin{array}{l}\text { T1-2 } 88 \% \\
>\text { T2 } 12 \%\end{array}$ & $\begin{array}{l}72 \\
-\end{array}$ & rT1-2 50 & & 50 & \\
\hline $\begin{array}{c}\text { Chen (2009) } \\
{[39 \bullet]}\end{array}$ & Endoscopic & 37 & & $\mathrm{~T} 1-3$ & 97 & $86(2 y r)$ & & $84(2 y r)$ & $\begin{array}{l}\text { No severe side } \\
\text { effects }\end{array}$ \\
\hline
\end{tabular}

$\mathrm{N}$, number of patients; CR, complete response; DFS, disease-free survival; OS, overall survival; LPFS, local progression-free survival

${ }^{*}$ No intracranial, skull base, or carotid extension. Most tumors were $<2 \mathrm{~cm}$

${ }^{* *}$ Only rT1-2 and including the "clear and close" margins. The authors do not explain the meaning of this term

- Vlantis et al. [38] retrospectively reviewed 80 patients (14 residual disease), all treated with curative intent. The majority $(95 \%)$ had rT1-2 stage. The transpalatal, the maxillary swing, and the midfacial degloving approach were compared. Of the rT1-2 tumors, clear pathological margins were achieved in $51 \%$, close margins in $18 \%$, and $31 \%$ had positive margins (with no differences between the approaches). If resection margins were positive, patients were treated with brachytherapy. The 5-year local control rate for rT1-2 was significantly better for the maxillary swing approach than the facial degloving approach ( $88 \%$ vs. $50 \%$ ). Because of the retrospective character of this study, there might have been a bias in tumor selection for both approaches. Survival was equal for the three different approaches.

- Recently, endoscopic surgery is introduced. Chen et al. [39•] treated 37 patients with tumors confined to the nasopharyngeal cavity, nasal septum, superficial parapharyngeal space, or the base of the sphenoid sinus. Radical margins were achieved in $97 \%$. During followup, no recurrences were seen for the rT1, but $29 \%$ of the tumors with parapharyngeal extension recurred. The authors concluded that the distance between the tumor and the carotid artery should be $>1 \mathrm{~cm}$ to achieve an adequate tumor margin.

\section{Complications}

- Wei et al. [37•] reported no mortality. Hospital admission ranged from 6 days to 4 months (median 13 days). Carotid artery bleeding 
was seen in three patients. Palatal fistula were seen in $20 \%$ and only occurred in patients with a midline mucosal incision. Only $6(2 \%)$ patients needed surgical repair. Trismus $<2 \mathrm{~cm}$ was seen in $21 \%$. Vlantis et al. [38] did not describe side effects. Chen's patients were discharged after 5 days. No severe complications were seen [39•].

\section{Special points}

- Positive resection margins and large tumor volume adversely influence local control and overall survival. Additional treatment with chemotherapy or radiation should be considered for these cases. Frozen sections are advisable during surgery. Before surgery, the surgeon must carefully estimate the likelihood of adequate resection margins. Tumors with intracranial extension or carotid artery involvement are not resectable $[37 \bullet, 38,39 \bullet]$.

\section{Photodynamic therapy}

\section{Procedure}

- Photodynamic therapy (PDT) for NPC is based on the intravascular injection of a photosensitizing drug. The tumor is then illuminated with a nonthermic laser, which leads to the production of active oxygen radicals, eventually causing local cell death. The effective treatment depth of PDT with second-generation photosensitizers is $10 \mathrm{~mm}$. With a special applicator, the nasopharynx can be illuminated uniformly with protection of the soft palate. Usually one treatment is adequate, which can be performed in an outpatient clinic under local anesthesia. Because there is no cumulative effect, PDT can be repeated. The procedure is easy to learn and does not need expensive equipment. These advantages make this modality very suitable for hospitals with limited radiation or surgery facilities (Table 3 ).

- Nyst et al. [17•] treated 22 patients with temoporfin (second-generation photosensitizer) mediated PDT in Indonesia. They did not

\begin{tabular}{|c|c|c|c|c|c|c|c|c|}
\hline PDT & Treatment & $\begin{array}{l}\text { No. of } \\
\text { patients }\end{array}$ & $\begin{array}{l}\text { Initial } \\
\text { T stage }\end{array}$ & Recurrent T stage & CR & DFS & OS & Side effects \\
\hline $\begin{array}{l}\text { Nyst (2012) } \\
{[17 \bullet]}\end{array}$ & PDT & 22 (21 residual) & & $\mathrm{T} 1 / 2<10$-mm depth & $100^{*}$ & & 55 (3 yr) & $\begin{array}{l}\text { Grade } 3 \text { : Headache } 32 \% \text {, } \\
\text { tinnitus } 5 \% \text {, myositis } \\
\text { neck } 5 \%\end{array}$ \\
\hline $\begin{array}{l}\text { Kulapaditharom } \\
\text { (1999) [40] }\end{array}$ & PDT & 13 (8 residual) & & $\begin{array}{l}\text { T1-2 } 46 \% \\
\text { T3-4 } 54 \%\end{array}$ & $\begin{array}{l}100 \\
14^{* *}\end{array}$ & & & \\
\hline $\begin{array}{l}\text { Lofgren (1995) } \\
\quad[41]\end{array}$ & PDT & 5 (3 residual) & T1-2 & $<10$-mm depth & & 60 (4 yr) & $60(4 \mathrm{yr})$ & $\begin{array}{l}\text { Headache } 100 \% \text {, minor } \\
\text { photosensitivity } 40 \%\end{array}$ \\
\hline $\begin{array}{l}\text { Indrasari } \\
\text { (2012) [42] }\end{array}$ & PDT & 1 & & T4 & & & $100(5 \mathrm{yr})$ & \\
\hline
\end{tabular}

CR, complete response; DFS, disease-free survival; OS, overall survival

${ }^{*}$ Only 17/22pts had tumor evaluation, all had CR

${ }^{* *}$ One patient had complete response, this patient also received chemotherapy 
make a distinction in therapy response between residual and recurrent disease, but only one patient had recurrent disease. All of the 17 evaluable patients had tumor-negative biopsies after PDT and the overall 3-year survival was $55 \%$ (27 \% died related to NPC).

- Kulapaditharom et al. [40] and Lofgren et al. [41] used hematoporphyrin (first-generation photosensitizer) mediated PDT and also had a complete response in all patients with T1-2 disease and a thickness less than $1 \mathrm{~cm}$ $(n=11)$. Patients with more extended disease had a partial response.

- T3-4 tumors are treated with a palliative intent only, because complete illumination of the tumor is in the majority not feasible. Nevertheless, treatment results are promising. In a case report, an intracranial extended tumor was treated by PDT and after 5 years this patient is still alive in good condition with no evidence of disease [42]. Also, Kulapaditharom's patients with T3-4 $(n=7)$ had considerable tumor regression of which five are still alive at 9, 16, 23, 37, and 40 months [40].

\section{Complications}

- In the weeks after the injection, the patient can gradually return to normal daylight exposure. Headache (grade 3) was seen in $33 \%$ in Nyst's study and disappeared in all patients. Kulapaditharom et al. did not describe the side effects. The patients described by Lofgren et al. all had headache, which was relieved by ordinary analgesics and disappeared at 38 months. Furthermore, middle ear effusion and tinnitus (grade 1-2) were frequently seen side effects. Hemorrhages were not reported.

\section{Special points}

- Nyst's study reveals the high incidence of residual disease in Indonesia, where NPC is one of the most frequent cancer types and where only limited access to radiotherapy and surgery facilities is available. NPC has a high incidence in many more countries where equal or even less facilities are available, such as North Africa or the remote regions in China. This stresses the need for more studies on residual disease and treatment modalities, which are easy to perform, low priced, and have a low complication rate.

\section{Chemotherapeutic and molecular treatment}

- Because small, local, residual lesions can be treated with good curative prognosis, chemotherapeutic and molecular regiments are only indicated when the lesions are too extended for surgery, brachytherapy, PDT, external beam, or stereotactic radiation. These palliative intent treatments fall beyond the scope of this article and will be discussed only briefly. Chemotherapeutic agents can be used in combination with the other modalities or as a single modality. NPC is sensitive to cisplatin-based regimens, with an overall response rate in patients with recurrent disease between $50 \%$ and $80 \%$, a median time to progression of 5-11 months and a median survival of 12- 
20 months [43-46]. Because patients with residual disease just finished treatment with cisplatin, the preferential choice might be a nonplatinum compound, such as a taxane, gemcitabine, or capecitabine. As monotherapy, these drugs induce response rates between $23 \%$ and $48 \%[3 \bullet, 47 \bullet, 48,49]$. More recently, studies have been conducted with monoclonal antibodies against epithelial growth factor (EGFR), although after chemotherapy failures. The results are not very satisfactory, with overall response rates of $11 \%$ and stable diseases in $20-48 \%$ for a limited period of time. A phase II study with the EGFR inhibitor gefitinib, after two lines of chemotherapy, in 19 patients with recurrent or metastatic NPC, resulted in no objective responses [50]. Also studies using EBV as target are conducted [51]. All studies included patients with failure on sides other than local, so the use of chemotherapy for isolated local failure has not been well studied yet.

\section{Conflict of Interest}

S.D. Stoker, J.N.A. van Diessen, J.P. de Boer, B. Karakullukcu, C.R. Leemans, and I.B. Tan declare they have no conflict of interest.

Human and Animal Rights and Informed Consent

This article does not contain any studies with human or animal subjects performed by any of the authors.

\section{Open Access}

This article is distributed under the terms of the Creative Commons Attribution License which permits any use, distribution, and reproduction in any medium, provided the original author(s) and the source are credited.

\section{References and Recommended Reading}

Papers of particular interest, published recently, have been highlighted as:

- Of importance

$\bullet \quad$ Of major importance

1. Chua DT, Sham JS, Hung KN, et al. Stereotactic radiosurgery as a salvage treatment for locally persistent and recurrent nasopharyngeal carcinoma. Head Neck. 1999;21(7):620-6.

2. Yu KH, Leung SF, Tung SY, et al. Survival outcome of patients with nasopharyngeal carcinoma with first local failure: a study by the Hong Kong Nasopharyngeal Carcinoma Study Group. Head Neck. 2005;27(5):397-405.
3. Wei WI, Kwong DL. Recurrent nasopharyngeal carcinoma: surgical salvage vs. additional chemoradiation. Curr Opin Otolaryngol Head Neck Surg. 2011;19(2):82-6.

Recently published article, considering the problem of local failures of nasopharyngeal carcinoma and the treatment modalities.

4. Yau TK, Sze WM, Lee WM, et al. Effectiveness of brachytherapy and fractionated stereotactic 
radiotherapy boost for persistent nasopharyngeal carcinoma. Head Neck. 2004;26(12):

1024-30.

5. Zheng XK, Chen LH, Chen YQ, Deng XG. Three-dimensional conformal radiotherapy versus intracavitary brachytherapy for salvage treatment of locally persistent nasopharyngeal carcinoma. Int J Radiat Oncol Biol Phys. 2004;60(1):165-70.

6. Leung TW, Tung SY, Sze WK, et al. Salvage brachytherapy for patients with locally persistent nasopharyngeal carcinoma. Int J Radiat Oncol Biol Phys. 2000;47(2):405-12.

7. Teo P, Leung SF, Choi P, Lee WY, Johnson PJ. Afterloading radiotherapy for local persistence of nasopharyngeal carcinoma. Br J Radiol. 1994;67(794):181-5.

8. Leung TW, Tung SY, Sze WK, et al. Treatment results of 1070 patients with nasopharyngeal carcinoma: an analysis of survival and failure patterns. Head Neck. 2005;27(7):555-65.

9. Teo PM, Kwan WH, Yu P, et al. A retrospective study of the role of intracavitary brachytherapy and prognostic factors determining local tumour control after primary radical radiotherapy in 903 non-disseminated nasopharyngeal carcinoma patients. Clin Oncol (R Coll Radiol). 1996;8(3):160-6.

10. Lee AW, Law SC, Foo W, et al. Nasopharyngeal carcinoma: local control by megavoltage irradiation. Br J Radiol. 1993;66(786):528-36.

11. Yan JH, Qin DX, Hu YH, et al. Management of local residual primary lesion of nasopharyngeal carcinoma (NPC): are higher doses beneficial? Int J Radiat Oncol Biol Phys. 1989;16(6):1465-9.

12. Chua DT, Sham JS, Kwong PW, Hung KN, Leung LH. Linear accelerator-based stereotactic radiosurgery for limited, locally persistent, and recurrent nasopharyngeal carcinoma: efficacy and complications. Int J Radiat Oncol Biol Phys. 2003;56(1):177-83.

13. Kwong DL, Wei WI, Cheng AC, et al. Long term results of radioactive gold grain implantation for the treatment of persistent and recurrent nasopharyngeal carcinoma. Cancer. 2001;91(6):1105-13.

14. Low JS, Chua ET, Gao F, Wee JT. Stereotactic radiosurgery plus intracavitary irradiation in the salvage of nasopharyngeal carcinoma. Head Neck. 2006;28(4):321-9.

15. Tanvetyanon T, Padhya T, McCaffrey J, et al. Prognostic factors for survival after salvage reirradiation of head and neck cancer. J Clin Oncol. 2009;27(12):1983-91.

16.• Wildeman MA, Fles R, Herdini C, et al. Primary treatment results of Nasopharyngeal Carcinoma (NPC) in Yogyakarta, Indonesia. PLoS One. 2013;8(5):e63706.

Recently published article, considering the problem of residual nasopharyngeal carcinoma disease in a high endemic country.
17. Nyst HJ, Wildeman MA, Indrasari SR, et al. Temoporfin mediated photodynamic therapy in patients with local persistent and recurrent nasopharyngeal carcinoma after curative radiotherapy: a feasibility study. Photodiagnosis Photodyn Ther. 2012;9(3):274-81.

Recently published article, considering the problem of local failures of nasopharyngeal carcinoma and the treatment modalities

18. Ferlay J, Shin HR, Bray F, et al. Estimates of worldwide burden of cancer in 2008: GLOBOCAN 2008. Int J Cancer. 2010;127(12):2893-917.

Recently published article, considering the problem of local failures of nasopharyngeal carcinoma and the treatment modalities.

19. Stoker SD, Wildeman MA, Fles R, et al. Prospective study: Current problems in radiotherapy for Nasopharyngeal Carcinoma in Yogyakarta, Indonesia. Presented at the NPC conference 2013. Istanbul, Turkey; June 20-22, 2013.

20. Wang WY, Twu CW, Lin WY, et al. Plasma Epstein-Barr virus DNA screening followed by (1)(8)F-fluoro-2-deoxy-D-glucose positron emission tomography in detecting posttreatment failures of nasopharyngeal carcinoma. Cancer. 2011;117(19):4452-9.

21. Adham M, Greijer AE, Verkuijlen SA, et al. EpsteinBarr virus DNA load in nasopharyngeal brushings and whole blood in nasopharyngeal carcinoma patients before and after treatment. Clin Cancer Res. 2013;19(8):2175-86.

22. Stevens SJ, Verkuijlen SA, Hariwiyanto B, et al. Noninvasive diagnosis of nasopharyngeal carcinoma: nasopharyngeal brushings reveal high Epstein-Barr virus DNA load and carcinoma-specific viral BARF1 mRNA. Int J Cancer. 2006;119(3):608-14.

23. Kwong DL, Nicholls J, Wei WI, et al. The time course of histologic remission after treatment of patients with nasopharyngeal carcinoma. Cancer. 1999;85(7):1446-53.

24. Hoebers F, Heemsbergen W, Moor S, et al. Reirradiation for head-and-neck cancer: delicate balance between effectiveness and toxicity. Int J Radiat Oncol Biol Phys. 2011;81(3):e111-8.

25. Chen AM, Phillips TL, Lee NY. Practical considerations in the re-irradiation of recurrent and second primary head-and-neck cancer: who, why, how, and how much? Int J Radiat Oncol Biol Phys. 2011;81(5):1211-9.

26. Law SC, Lam WK, Ng MF, et al. Reirradiation of nasopharyngeal carcinoma with intracavitary mold brachytherapy: an effective means of local salvage. Int J Radiat Oncol Biol Phys. 2002;54(4):1095-113.

27. Wu SX, Chua DT, Deng ML, et al. Outcome of fractionated stereotactic radiotherapy for 90 patients with locally persistent and recurrent nasopharyngeal carcinoma. Int J Radiat Oncol Biol Phys. 2007;69(3):761-9. 
28. Liu F, Xiao JP, Xu YJ, et al. Fractionated stereotactic radiotherapy with vagina carotica protection technique for local residual nasopharyngeal carcinoma after primary radiotherapy. Chin Med J (Engl). 2012;125(14):2525-9.

Recently published article, considering the problem of local failures of nasopharyngeal carcinoma and the treatment modalities.

29. Chua DT, Wu SX, Lee V, Tsang J. Comparison of single versus fractionated dose of stereotactic radiotherapy for salvaging local failures of nasopharyngeal carcinoma: a matched-cohort analysis. Head Neck Oncol. 2009;1:13.

30. Yan JH, Xu GZ, Hu YH, et al. Management of local residual primary lesion of nasopharyngeal carcinoma: II. Results of prospective randomized trial on booster dose. Int J Radiat Oncol Biol Phys. 1990;18(2):295-8.

31. Chua DT, Sham JS, Leung LH, Au GK. Re-irradiation of nasopharyngeal carcinoma with intensity-modulated radiotherapy. Radiother Oncol. 2005;77(3):290-4

32. Hua YJ, Han F, Lu LX, Mai HQ, et al. Long-term treatment outcome of recurrent nasopharyngeal carcinoma treated with salvage intensity modulated radiotherapy. Eur J Cancer. 2012;48(18):3422-8.

33. Qiu S, Lin S, Tham IW, Pan J, Lu J, Lu JJ. Intensitymodulated radiation therapy in the salvage of locally recurrent nasopharyngeal carcinoma. Int J Radiat Oncol Biol Phys. 2012;83(2):676-83.

Recently published article, considering the problem of local failures of nasopharyngeal carcinoma and the treatment modalities.

34. Xiao J, Xu G, Miao Y. Fractionated stereotactic radiosurgery for 50 patients with recurrent or residual nasopharyngeal carcinoma. Int J Radiat Oncol Biol Phys. 2001;51(1):164-70.

35. Gondhowiardjo S, Prajogi G, Sekarutami S. History and growth of radiation oncology in Indonesia. Biomed Imaging Interv J. 2008;4(3):e42.

36. Slotman BJ, Cottier B, Bentzen SM, et al. Overview of national guidelines for infrastructure and staffing of radiotherapy. ESTRO-QUARTS: work package 1. Radiother Oncol. 2005;75(3):349-54.

37. Wei WI, Chan JY, Ng RW, Ho WK. Surgical salvage of persistent or recurrent nasopharyngeal carcinoma with maxillary swing approach - Critical appraisal after 2 decades. Head Neck. 2011;33(7):96975 .

Recently published article, considering the problem of local failures of nasopharyngeal carcinoma and the treatment modalities.

38. Vlantis AC, Yu BK, Kam MK, Hung T, et al. Nasopharyngectomy: does the approach to the nasopharynx influence survival? Otolaryngol Head Neck Surg. 2008;139(1):40-6.
39.• Chen MY, Wen WP, Guo X, et al. Endoscopic nasopharyngectomy for locally recurrent nasopharyngeal carcinoma. Laryngoscope. 2009;119(3):516-22.

Recently published article, considering the problem of local failures of nasopharyngeal carcinoma and the treatment modalities.

40. Kulapaditharom B, Boonkitticharoen V. Photodynamic therapy for residual or recurrent cancer of the nasopharynx. J Med Assoc Thai. 1999;82(11):1111-7.

41. Lofgren LA, Hallgren S, Nilsson E, et al. Photodynamic therapy for recurrent nasopharyngeal cancer. Arch Otolaryngol Head Neck Surg. 1995;121(9):997-1002.

42. Indrasari SR, Timmermans AJ, Wildeman MA, et al. Remarkable response to photodynamic therapy in residual T4N0M0 nasopharyngeal carcinoma: a case report. Photodiagnosis Photodyn Ther. 2012;9(4):319-20.

43. Chan AT. Nasopharyngeal carcinoma. Ann Oncol. 2010;21 Suppl 7:vii308-12.

44. Boussen H, Cvitkovic E, Wendling JL, et al. Chemotherapy of metastatic and/or recurrent undifferentiated nasopharyngeal carcinoma with cisplatin, bleomycin, and fluorouracil. J Clin Oncol. 1991;9(9):1675-81.

45. Taamma A, Fandi A, Azli N, et al. Phase II trial of chemotherapy with 5-fluorouracil, bleomycin, epirubicin, and cisplatin for patients with locally advanced, metastatic, or recurrent undifferentiated carcinoma of the nasopharyngeal type. Cancer. 1999;86(7):1101-8.

46. Ngan RK, Yiu HH, Lau WH, et al. Combination gemcitabine and cisplatin chemotherapy for metastatic or recurrent nasopharyngeal carcinoma: report of a phase II study. Ann Oncol. 2002;13(8):1252-8.

47. Bensouda Y, Kaikani W, Ahbeddou N, et al. Treatment for metastatic nasopharyngeal carcinoma. Eur Ann Otorhinolaryngol Head Neck Dis. 2011;128(2):79-85.

Recently published article, considering the problem of local failures of nasopharyngeal carcinoma and the treatment modalities.

48. Foo KF, Tan EH, Leong SS, et al. Gemcitabine in metastatic nasopharyngeal carcinoma of the undifferentiated type. Ann Oncol. 2002;13(1):150-6.

49. Chua D, Wei WI, Sham JS, Au GK. Capecitabine monotherapy for recurrent and metastatic nasopharyngeal cancer. Jpn J Clin Oncol. 2008;38(4):244-9.

50. Chua DT, Wei WI, Wong MP, et al. Phase II study of gefitinib for the treatment of recurrent and metastatic nasopharyngeal carcinoma. Head Neck.

2008;30(7):863-7.

51. Wildeman MA, Novalic Z, Verkuijlen SA, et al. Cytolytic virus activation therapy for Epstein-barr virus driven tumours. Clin Cancer Res.

2012;18(18):5061-70. 